\title{
Mechanism of Autophagy Regulation in
}

\section{MPTP-Induced PD Mice via the mTOR Signaling Pathway by Echinacoside}

\author{
Zhen-Nian Zhang ${ }^{\text {I,* }}$ \\ Zhen Huil,* \\ Chang Chen' \\ Yan Liang' \\ Li-Li Tang \\ Su-Lei Wang' \\ Cheng-Cheng $\mathrm{Xu}{ }^{\prime}$ \\ Hui Yang' \\ Yang Zhao' \\ Jing-Si Zhang ${ }^{2}$
}

'Department of Neurology, Nanjing Hospital of Chinese Medicine Affiliated to Nanjing University of Chinese Medicine, Nanjing, 2 I0022, People's Republic of

China; ${ }^{2}$ Department of Neurology, Shuguang Hospital Affiliated to Shanghai University of Traditional Chinese Medicine, Shanghai, 201203, People's Republic of China

*These authors contributed equally to this work
Correspondence: Jing-Si Zhang Department of Neurology, Shuguang Hospital Affiliated to Shanghai University of Traditional Chinese Medicine, No. 528 of Zhangheng Road, Pudong New Area, Shanghai, 201203, People's Republic of China

Tel/Fax +86 I3 I 27823579

Email zhangzn88_dr@163.com
Objective: The present study aimed to investigate the effect of echinacoside on autophagyrelated indicators through the $\mathrm{mTOR}$ signaling pathway, especially the effect on the clearance of autophagy substrate P62 and $\alpha$-synuclein, the core pathological products of Parkinson's disease (PD), to provide new strategies for the treatment of PD.

Methods: A mouse model of subacute PD was established by the intraperitoneal injection of 1-methyl-4-phenyl-1,2,3,6-tetrahydropyridine (MPTP). First, the neurobehavioral symptoms in mice of each group were evaluated, and the monoamine neurotransmitters in the striatum in each group were measured with a high-performance liquid phase. Immunofluorescence double staining was adopted to observe the expression of tyrosine hydroxylase (TH), $\alpha$ synuclein, and LC3. The transmission electron microscope was used to observe the changes of ultrastructure in substantia nigra and the formation of autophagosomes. Then, the expressions of TH, $\alpha$-synuclein, Beclin 1, LC3, P62, mTOR, and the up-stream protein AKT were detected by Western blot.

Results: When compared with the model group, the neurobehavioral function significantly improved in the echinacoside group $(\mathrm{P}<0.01)$, together with increased expression of $\mathrm{TH}$, $\mathrm{DA}$, and DOPAC in the brain $(\mathrm{P}<0.01)$. In the echinacoside group, while the expressions of Beclin 1 and LC3-II increased $(\mathrm{P}<0.01)$, the expression levels of P62 and $\alpha$-synuclein decreased significantly $(\mathrm{P}<0.01)$. Echinacoside could up-regulate the expression level of the survival signal p-AKT/AKT and decrease the expression of mTOR.

Conclusion: Echinacoside could increase autophagy by inhibiting the expression of mTOR, thereby promoting the clearance of $\alpha$-synuclein and the degradation of the autophagy substrate P62 and exerting the neuroprotective effect.

Keywords: Parkinson's disease, MPTP, echinacoside, $\alpha$-synuclein, P62, autophagy, mTOR

\section{Introduction}

Parkinson's disease (PD) is a chronic and progressive neurodegenerative disease. A typical pathological feature of PD is the formation of Lewy bodies, and $\alpha$ synuclein and ubiquitin are the main components of the Lewy bodies. ${ }^{1,2}$

More and more evidence shows that the disorder of autophagy regulation eventually leads to the accumulation of misfolded proteins and injured organelles. $^{3,4}$ The autophagy-lysosome pathway can not only degrade proteins that cannot be degraded by the ubiquitin-proteasome pathway but also the pathway can degrade $\alpha$-synuclein. ${ }^{5,6}$ Therefore, the regulation of autophagy may provide a new strategy for the treatment of PD. Autophagy is the main pathway 
for the degradation of intracellular aggregates, and mTOR kinase is a key regulatory site for autophagy. Therefore, it is of theoretical and practical significance to elucidate the relationship between PD and autophagy regulated by the mTOR signaling pathway. Since the degradation of $\alpha$-synuclein mainly depends on the autophagy-lysosomal system with the elimination of the abnormal proteins as the target, the development of neuroprotective drugs able to regulate autophagy may provide directions for the treatment of neurodegenerative diseases. $^{7}$ Echinacoside $(\mathrm{ECH})$ has a molecular formula of $\mathrm{C}_{35} \mathrm{H}_{46} \mathrm{O}_{20}$. It is the main component of the Chinese medicine Cistanche phenylethanol glycosides. It was first isolated by Stoll et al from the root of the Echinacea Angustifolia (or Echinacea Angustifolia) DC. ${ }^{8}$ ECH has a wide range of pharmacological effects. It has antiinflammatory, anti-oxidant, nerve protection, improvement of learning and memory, hepatic protection, immune regulation, and anti-tumor effects. ${ }^{9}$

The present study, starting from the core pathological mechanisms of PD, aimed to investigate the regulation of the neuroprotective effects of echinacoside in a mouse PD model induced by MPTP from the autophagy-lysosomal pathway.

\section{Materials and Apparatus Experimental Animal}

The experimental group consisted of 108 male C57BL/6J mice with the SPF grade, eight weeks old, weighing 20-22 g, four mice per cage, kept at $22-25^{\circ} \mathrm{C}$. The mice were provided by Shanghai Center for Laboratory Animals, Chinese Academy of Sciences, housed at Nanjing University of Chinese Medicine, and animals received humane care according to the National Institutes of Health guidelines, in the SPF grade room with a temperature of $22-25{ }^{\circ} \mathrm{C}$, a relative humidity of $55 \%$, and under the 12-hour circadian rhythm. The mice were free to have food and water.

\section{Main Reagents}

MPTP (Sigma Company), mice anti -TH monoclonal antibody (Sigma Company), Alexa Fluor 555 anti-rabbit antibody (Biyuntian Institute of Biotechnology), Alexa Fluor 488 anti-rabbit antibody (Biyuntian Institute of Biotechnology), HRP-goat anti-rabbit secondary antibody (Biyuntian Institute of Biotechnology), rabbit polyclonal $\alpha$ synuclein antibody (Cell Signaling Technology Company), HRP-goat anti-mice secondary antibody (Biyuntian Institute of Biotechnology), echinacoside (Chengdu Linghangzhe
Biotechnology Co., Ltd.), rabbit anti-p-AKT (Ser473) antibody (Cell Signaling Technology Company), Rapamycin (Cell Signaling Technology Company), Chloroquine (Sigma Company), Wortmannin (Sigma Company), rabbit polyclonal LC3 antibody (Abcam Company), rabbit polyclonal P62 antibody (Enzo Life Science Company), mice monoclonal $\beta$-actin antibody (Santa Cruz Company), Beclin 1 polyclonal antibody (Abcam Company).

Information of the materials: MPTP (America, Sigma Company),mice anti TH monoclonal antibody (America, Sigma Company), rabbit polyclonal $\alpha$-synuclein antibody (America, Cell Signaling Technology Company), HRP-goat anti-mice secondary antibody (China, Biyuntian Institute of Biotechnology), echinacoside (China, Chengdu Linghangzhe Biotechnology Co., Ltd.), rabbit anti-p-AKT (Ser473) antibody (America, Cell Signaling Technology Company), Rapamycin (America, Cell Signaling Technology Company), Chloroquine (America, Sigma Company), Wortmannin (America,Sigma Company), rabbit polyclonal LC3 antibody (America, Abcam Company), rabbit polyclonal P62 antibody (America, Enzo Life Science Company), mice monoclonal $\beta$ actin antibody (America, Santa Cruz Company), Beclin 1 polyclonal antibody (America, Abcam Company).

\section{Experimental Methods Grouping of the Experimental Animals and Medication Grouping of Experimental Animals}

The $108 \mathrm{C} 57 \mathrm{BL} / 6 \mathrm{~J}$ mice were randomly divided into six groups, with 18 mice in each group. The details were as follows: The normal control group (the normal saline group, $\mathrm{N}$ group), the model group (MPTP group, $\mathrm{M}$ group), the echinacoside group (MPTP + echinacoside, EH group), the induced autophagy group (MPTP + Rapamycin group, RA group), the autophagy inhibitor A group (MPTP + echinacoside + chloroquine group, CQ group), the autophagy inhibitor B group (MPTP + echinacoside + Wortmannin group, WO group). Dose of MPTP was $30 \mathrm{mg} / \mathrm{kg} / \mathrm{d}$,continuously injected for 7 days. Echinacoside was given at $30 \mathrm{mg} / \mathrm{kg} / \mathrm{d}$, intragastrically administrated. ${ }^{9}$

\section{Modeling of the Experimental Animals and Medication}

The $\mathrm{N}$ group and $\mathrm{EH}$ group were given normal saline and echinacoside three days before modeling, respectively, to seven days after modeling. Rapamycin, ${ }^{10}$ wortmannin, ${ }^{11}$ and chloroquine were administered for seven consecutive days after the last injection of MPTP. The dose of tail vein 
injection of rapamycin was calculated as $2 \mathrm{mg} / \mathrm{kg} / \mathrm{d}$. The dose of intraperitoneal injection of chloroquine in mice was $50 \mathrm{mg} / \mathrm{kg} / \mathrm{d}$. The dose of tail vein injection of wortmannin in mice was $0.7 \mathrm{mg} / \mathrm{kg} / \mathrm{d}$ (Supplemental Figure 1).

\section{The Neurobehavioral Observations The Pole Test}

According to the literature, ${ }^{12}$ with minor modification, the self-made device for the pole test was as follows: The device was a $55 \mathrm{~cm}$ long, $1 \mathrm{~cm}$ in diameter PV tube with a diameter of $2 \mathrm{~cm}$ spherical projection at the top of the device as an attachment point for mice. The device was covered with black tape to prevent the mice from falling. Each mouse was placed on the spherical protruding point with its head upward during the test. The time from the top of the placed bar to the head to turn down (T-turn) and the total time from the top of the placed bar to climb to the bottom of the tube to land (T-total) were recorded.

\section{The Spontaneous Activity}

The spontaneous activity, also known as the open field activity, is a common indicator for detecting the rare activity after MPTP injury. ${ }^{13}$ In the present study, an open field experiment video analysis system was adopted. The specific operations were as follows: Before the experiment, four mice of the same group were put into an observation box (with a length of $25 \mathrm{~cm}$, a width of $25 \mathrm{~cm}$, and a height of $25 \mathrm{~cm}$ ) to adapt for 30 minutes. They were then video recorded continuously for 30 minutes. In the 30-minute observation time window, the number of vertical movements (Rearing number) was counted separately in three discontinuous intervals of five minutes. The video analysis system automatically analyzed the trajectory of the mouse's activities and obtained a 30-minute total distance (Total Distance) as an indicator of horizontal movement.

\section{The Gait Analysis}

Gait analysis: According to the literature, ${ }^{14}$ a self-made gait analysis device was used. This device was as follows: A wooden walkway (with a length of $42 \mathrm{~cm}$, a width of $4.5 \mathrm{~cm}$, and a height of $12 \mathrm{~cm}$ ), one end was open, the other end led to the squirrel cage. The squirrel cage was covered with a black cloth. Blue ink was dipped on the bottom of the mouse's feet. Then the mouse was placed on the open end of the walkway. The mouse naturally moved to the end of the squirrel cage that was covered with black cloth. The three longest fore and hind limb strides were measured respectively, and the average values were taken.

\section{The Rotarod Test}

The mouse was placed on a variable-speed rotarod, with the speed of the rotarod being adjusted to increase from four rounds/minute to 40 rounds/minute within five minutes. The time from the beginning of the rotarod rotation to the mouse's drop from the rotarod each time was recorded as latency to fall. ${ }^{15}$

\section{Determination of the Contents of Monoamine Neurotransmitters in the Striatum by High-Performance Liquid Chromatography Electrochemistry Detection (HPLC-ECD)}

\section{Sampling and Treatment}

The mice were euthanized, and the striatum was quickly separated on ice and weighed. According to the ratio of $1 \mathrm{~mL}$ of $5 \%$ perchloric acid to $0.1 \mathrm{~g}$ of the brain (wet weight), each group's specimens were put into the glass containing the corresponding volume of perchloric acid and homogenized in a slurry tube. The homogenate was centrifuged at a low temperature with 13,000 r/min for 20 minutes. The supernatant was taken, and the centrifugation was repeated. The supernatant was placed in a low-temperature refrigerator for further detection.

\section{The Conditions for HPLC-ECD Were as Follows}

The Couloehemlll 5300 electrochemical chromatography was adopted. The detection means were 5020 and $504 \mathrm{lb}$ cells, with the chromatographic column of MD-150 $(3.0 * 150 \mathrm{~mm} ; 3 \mathrm{um})$, the mobile phase of MDTM-2, and the sensitivity of $2 \mathrm{uA}$. The flow rate was set to $0.6 \mathrm{~mL} /$ min, the potential of the electrode was set to $220 \mathrm{mV}$, and the column temperature was set to $30^{\circ} \mathrm{C}$. The injection volume was $20 \mathrm{ul}$ (first diluted ten times).

\section{The Contents of the Following Neurotransmitters Were Detected}

Dopamine (DA), Dihydroxyphenylacetic acid (DOPAC), High vanillic acid (HVA), Norepinephrine (NE), 5-Hydroxytryptamine (5-HT), 5-Hydroxyindoleacetic acid (5-HIAA).

\section{The Immunofluorescent Staining of $\mathrm{TH}$, $\alpha$-Synuclein, LC3 and p62}

The mice ( $\mathrm{n}=6$ per group) were euthanized under deep anesthesia (5\% chloral hydrate) and perfused with $0.9 \%$ 
sodium chloride, followed by $4 \%$ paraformaldehyde through the left cardiac ventricle. Brains were quickly removed and postfixed for 24 hours, then transferred to phosphate-buffered saline (PBS) containing 30\% sucrose at $4^{\circ} \mathrm{C}$ until they sank. Tissue was embedded in paraffin, and coronal sections $(30 \mu \mathrm{m})$ were cut through the substantia nigra pars compacta ( $\mathrm{SNc}$; -2.8 to $-3.8 \mathrm{~mm}$ caudal to the bregma) using a sledge microtome. The sections were dewaxed and hydrated, and the endogenous peroxidase was quenched with $0.3 \% \mathrm{H}_{2} \mathrm{O}_{2}$ for 30 minutes. After heat-induced antigen retrieval, the slides were incubated with $0.5 \%$ Triton-X100 then $5 \%$ bovine serum albumin for 30 minutes each. The sections were then incubated with anti- $\alpha$-synuclein antibody, anti-TH antibody, anti-LC3 antibody, anti-p62 antibody, and the corresponding fluorescent secondary antibodies overnight at $4^{\circ} \mathrm{C}$. After three five-minute washes in PBS, the sections were incubated in biotinylated secondary antibody for one hour, followed by avidin-biotin complex for 30 minutes. Peroxidase activity was visualized with 3.3-diaminobenzidine.

\section{The Detection of Protein by Western Blot}

$\mathrm{SNc}$ protein was extracted ( $\mathrm{n}=6$ mice per group), separated using $12-15 \%$ SDS-PAGE, and transferred to PVDF membranes. The membranes were blocked with 5\% nonfat milk for 1.5 hours at room temperature and incubated with antibodies against TH, $\alpha$-synuclein, p62, Beclin 1, LC3, p-AKT, AKT, p-mTOR, mTOR, and GAPDH at $4^{\circ} \mathrm{C}$ overnight. The next day, membranes were rinsed with tris-buffered saline containing $0.1 \%$ Tween 20 and hybridized with corresponding secondary antibodies for 1.5 hours. Proteins were visualized using enhanced chemiluminescence, and immunoreactive bands were analyzed with image analysis software (Gel Doc 1000-UV; Bio-Rad, Richmond, CA) to calculate the optical density of $\alpha$-synuclein and TH bands normalized to GADPH.

Information of antibodies: rabbit polyclonal LC3 antibody: 1:1000. Rabbit polyclonal P62 antibody: 1:1000. Beclin 1 polyclonal antibody: 1:1000. Mice anti TH monoclonal antibody: 1:1000. Rabbit polyclonal $\alpha$-synuclein antibody: 1:1000.

\section{Observation of the Ultrastructure of the Substantia Nigra by Transmission Electron Microscope}

Mice ( $\mathrm{n}=6$ per group) were euthanized 5\% chloral hydrate and perfused through the left ventricle with ice- cold PBS, followed by $4 \%$ paraformaldehyde and $1 \%$ glutaraldehyde in PBS. Brains were removed and postfixed in $2.5 \%$ glutaraldehyde at $4^{\circ} \mathrm{C}$ for six hours. The SNc was cut into blocks of about $1 \mathrm{~mm}^{3}$ and fixed in PBS containing $2.5 \%$ glutaraldehyde and preserved at $4^{\circ} \mathrm{C}$ for further processing. The fragments were postfixed in $1 \%$ osmium tetroxide in the same buffer, dehydrated through graded alcohols, and embedded in Epon 812 (Shell Chemical Co., Houston, TX). Ultrathin (50 nm) sections were cut using an ultramicrotome, stained using uranyl acetate and lead citrate, and analyzed under a transmission electron microscope (Leica TCS SP2CLSM, School of Medicine Electron Microscopy Center, Fudan University).

\section{Statistical Analysis}

All measurement data in the present study were expressed in the form of means \pm standard errors, and the statistical analysis was processed by the GraphPad Prism 5.0 (GraphPad Software Inc, San Diego, CA) software. P < 0.05 was considered statistically significant. One-way analysis of variance (one-way ANOVA) was used to test the statistical differences, and the comparison between multiple groups was analyzed by Tukey's multiple group comparisons.

\section{The Experimental Results} Improvement of the Neurobehavioral Symptoms in MPTP-Induced PD Mice by Echinacoside Echinacoside Shortened the Climbing Time in MPTP-Induced PD Mice

The T-turn and T-Total in the $\mathrm{M}$ group were noticeably longer than those in the $\mathrm{N}$ group $(\mathrm{P}<0.01)$. The T-turn and T-Total in the EH group significantly decreased compared with those in the $\mathrm{M}$ group $(\mathrm{P}<0.01)$. Compared with the $\mathrm{EH}$ group and $\mathrm{M}$ group, the above two indexes in the CQ group and WO group increased significantly $(\mathrm{P}<$ 0.01 and $\mathrm{P}<0.05$, respectively). Comparisons between the EH group and RA group showed there was no significant difference in T-turn and T-Total (as illustrated in Figure 1A).

Echinacoside Increased the Spontaneous Activity in MPTP-Induced PD Mice

The rearing number within five minutes, and the total distance within 30 minutes all significantly decreased in the $\mathrm{M}$ group compared with those in the $\mathrm{N}$ group $(\mathrm{P}<$ $0.01)$. The above two indexes significantly increased in the 
A
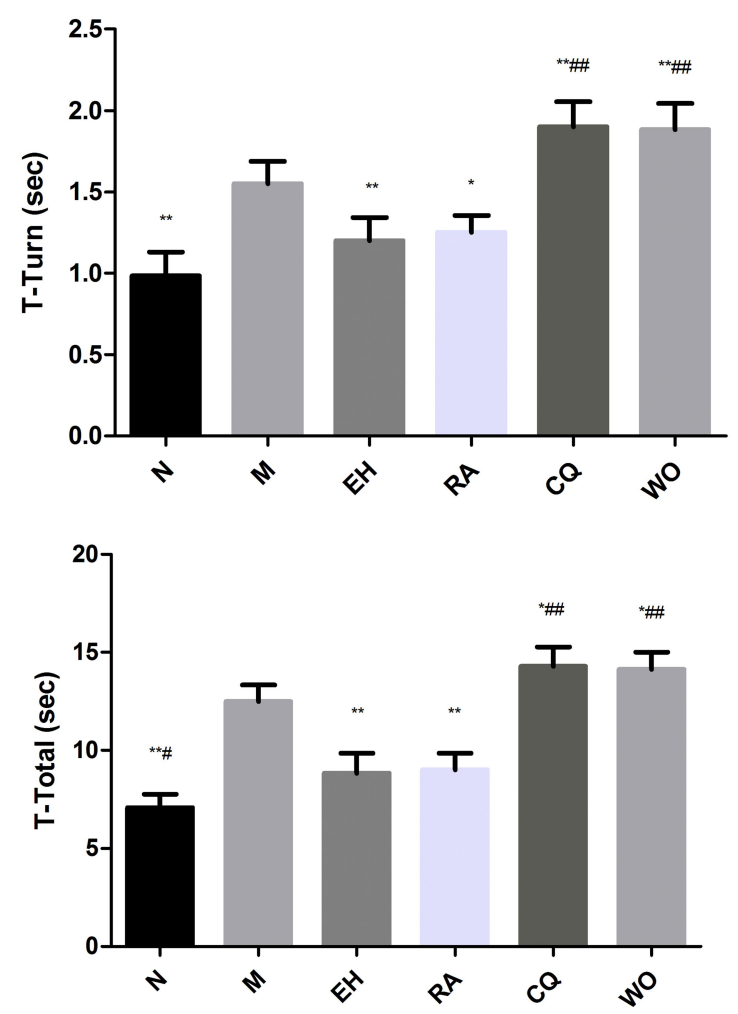

C
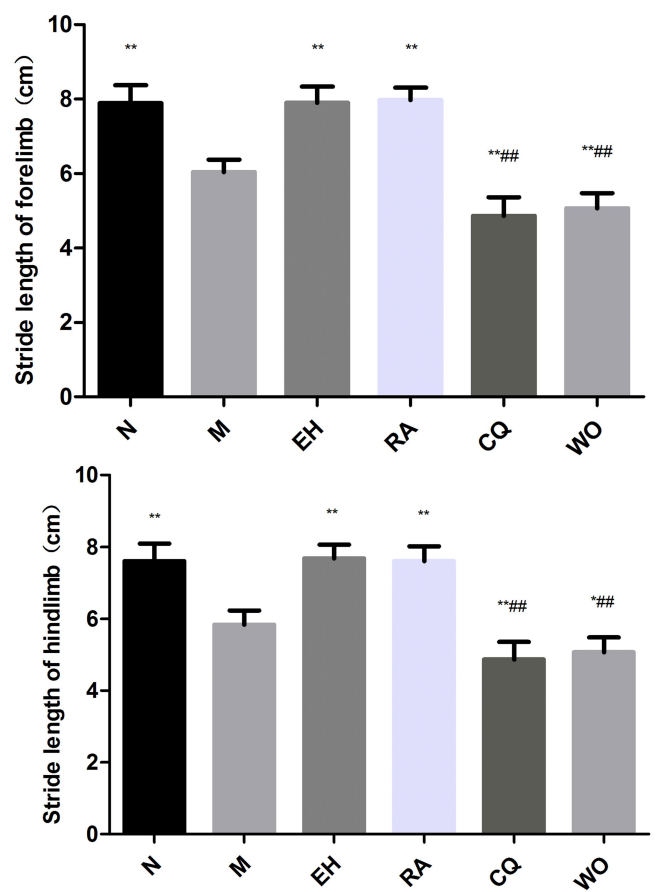

B
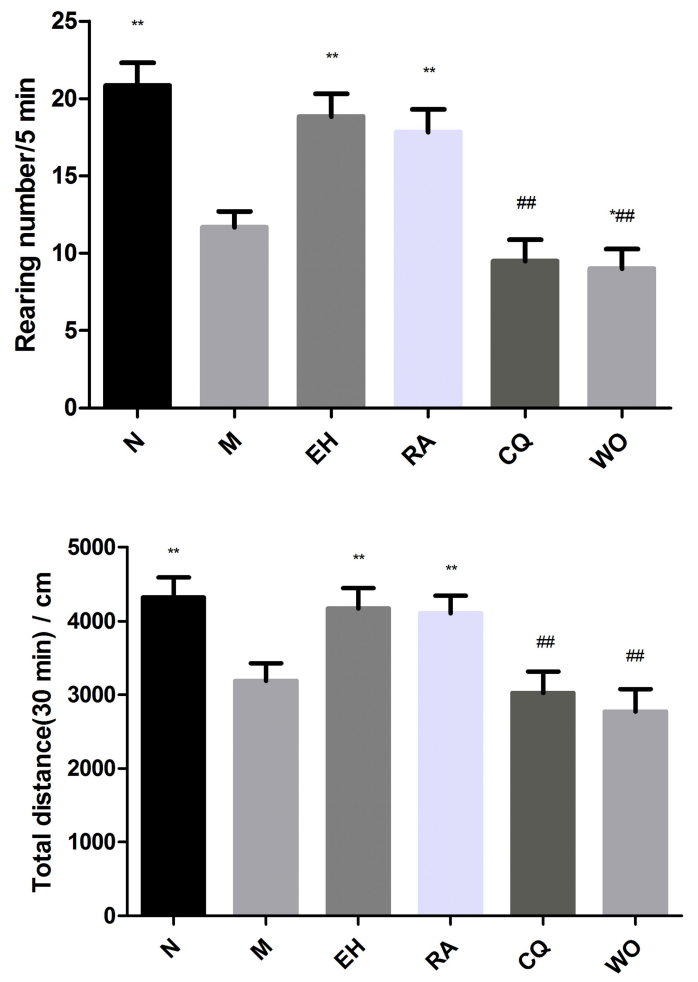

D

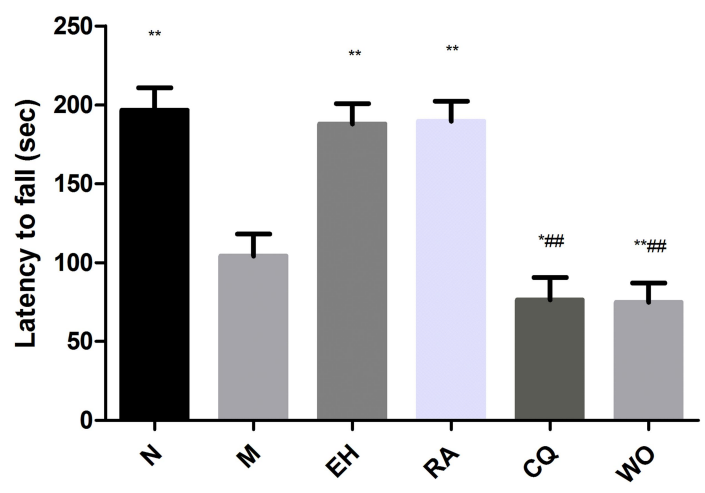

Figure I The neurobehavior in mice of each group. (A) The pole test in mice of each group: Turning time (T-Turn); Total climbing time (T-Total). (B) The spontaneous activity in mice of each group: The erection times within five minutes (Rearing number); The horizontal movement distance within 30 minutes (Total Distance). (C) The gait analysis in mice of each group: The stride length of fore limb; The stride length of the hind limb. (D) The rotarod in mice of each group: The staying time on the rotarod (also known as the latency to fall).

Notes: ${ }^{*} \mathrm{P}<0.05,{ }^{* *} \mathrm{P}<0.01$, compared with the model group; ${ }^{\# *} \mathrm{P}<0.01$, compared with the high-dose echinacoside group, $\mathrm{n}=6$. 
EH group and the RA group compared with those in the $\mathrm{M}$ group $(\mathrm{P}<0.01)$. Compared with the $\mathrm{EH}$ group, the rearing number and the total distance significantly decreased in the CQ group and the WO group $(\mathrm{P}<0.01)$. There were no statistically significant differences in the above two indexes between the EH group and RA group $(P>0.05)$ (as shown in Figure 1B).

\section{Echinacoside Prolonged the Fore and Hind Limb} Strides in MPTP-Induced PD Mice

The fore and hind limb strides significantly reduced in the $\mathrm{M}$ group compared with those in the $\mathrm{N}$ group $(\mathrm{P}<0.01)$. The fore and hind limb strides significantly increased in the EH group and RA group compared with those in the $\mathrm{M}$ group $(\mathrm{P}<0.01)$. Compared with the $\mathrm{EH}$ group and the $\mathrm{M}$ group, the fore and hind limb strides were significantly reduced in the CQ group and the WO group ( $\mathrm{P}<0.01$ and $\mathrm{P}<0.05$, respectively). There were no statistically significant differences in fore and hind limb strides between the EH group and RA group (as demonstrated in Figure 1C).

\section{Echinacoside Prolonged the Latency to Fall in MPTP-Induced PD Mice}

The staying time on the rotarod, also known as the latency to fall, significantly reduced in the $\mathrm{M}$ group compared with the $\mathrm{N}$ group $(\mathrm{P}<0.01)$. The latency to fall significantly increased in the $\mathrm{EH}$ group and the RA group compared with the $\mathrm{M}$ group $(\mathrm{P}<0.01)$. The latency to fall significantly reduced in the CQ group and WO group $(\mathrm{P}<0.01$ and $\mathrm{P}<0.05$, respectively) compared with the EH group and $\mathrm{M}$ group. There were no statistically significant differences in the latency to fall between the EH group and RA group $(\mathrm{P}>0.05)$ (as shown in Figure 1D).

\section{Echinacoside Increased the Content of DA and Metabolites in the Striatum in MPTP-Induced PD Mice}

Compared with the $\mathrm{N}$ group, the contents of DA, DOPAC, and HVA in the $\mathrm{M}$ group significantly reduced $(\mathrm{P}<0.01)$. The contents of DA, DOPAC, and HVA significantly increased in the EH group and the RA group compared with those in the $\mathrm{M}$ group $(\mathrm{P}<0.01)$. Compared with the EH group and $\mathrm{M}$ group, the contents of DA, DOPAC, and HVA significantly decreased in the CQ group and the WO group ( $\mathrm{P}<0.01$ and $\mathrm{P}<0.05$, respectively). There were no statistically significant differences in the contents of DA, DOPAC, and HVA between the EH group and the RA group $(\mathrm{P}>0.05)$ (as shown in Figure $2 \mathrm{~A}-\mathrm{C}$ ). Compared with the $\mathrm{N}$ group, the content of 5-HT significantly decreased in the $\mathrm{M}$ group $(\mathrm{P}<0.01)$, and the content of 5 -HT in the EH group significantly increased $(\mathrm{P}<0.01)$. Compared with the EH group, the content of 5-HT significantly decreased in the CQ group and the WO group ( $\mathrm{P}$ $<0.01$ ) (as demonstrated in Figure 2D).

\section{Echinacoside Increased the Expression of $\mathrm{TH}$ in the Substantia Nigra and Decreased the Expression of $\alpha$-Synuclein in PD Mice}

First, double immunofluorescence staining was conducted for TH and $\alpha$-synuclein. Compared with the $\mathrm{N}$ group, the expression of $\mathrm{TH}$ in the substantia nigra significantly reduced in the $\mathrm{M}$ group, while the expression of $\alpha$-synuclein significantly increased. However, compared with the $\mathrm{M}$ group, the expression of $\alpha$ synuclein significantly decreased in the EH group and the RA group. The expressions of $\alpha$-synuclein significantly increased in the CQ group and the WO group, which were even higher than that in the $M$ group (as demonstrated in Figure 3).

Then, Western blot was adopted to measure the above two indicators. The results showed that the protein expression of $\mathrm{TH}$ in the $\mathrm{M}$ group was significantly lower than that in the $\mathrm{N}$ group $(\mathrm{P}<0.01)$. The protein expressions of $\mathrm{TH}$ in the EH group and RA group significantly increased compared with that in the $\mathrm{M}$ group $(\mathrm{P}<0.01)$. The protein expressions of TH in the CQ group and WO group were significantly decreased compared with that in the $\mathrm{EH}$ group ( $\mathrm{P}<0.01)$. The protein expression of $\alpha$-synuclein in the $\mathrm{M}$ group also increased compared with the $\mathrm{N}$ group $(\mathrm{P}<0.01)$. However, the protein expressions of $\alpha$ synuclein in the EH group and the RA group significantly decreased compared with that in the $\mathrm{M}$ group, and the differences were statistically significant $(\mathrm{P}<0.01)$. Compared with the EH group and the $\mathrm{M}$ group, the protein expressions of $\alpha$-synuclein in the CQ group and the WO group increased significantly, and the differences were statistically significant $(\mathrm{P}<0.01)$ (as shown in Figure 4).

\section{Echinacoside Decreased the Expression of P62 in the Substantia Nigra in MPTP-Induced PD Mice}

First, Western blot was adopted to detect the protein expression of P62. The results revealed that the protein expression 
A

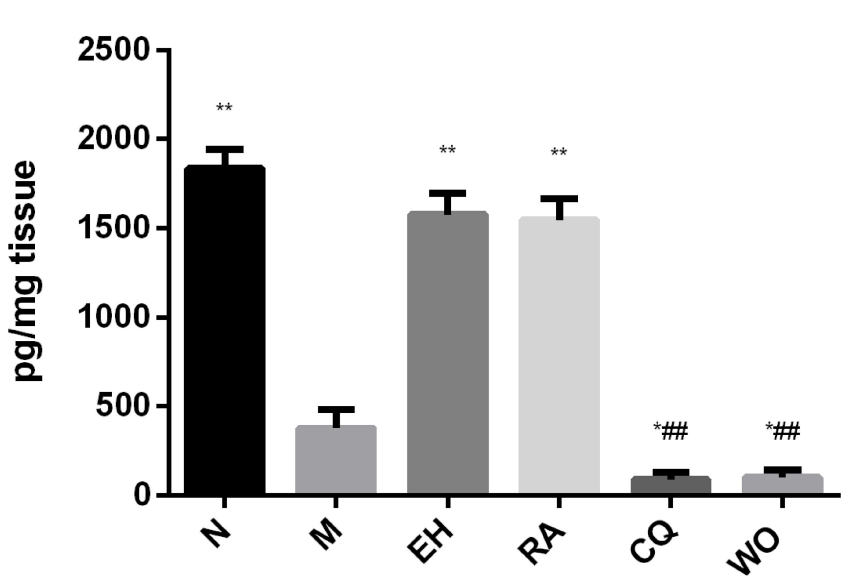

C

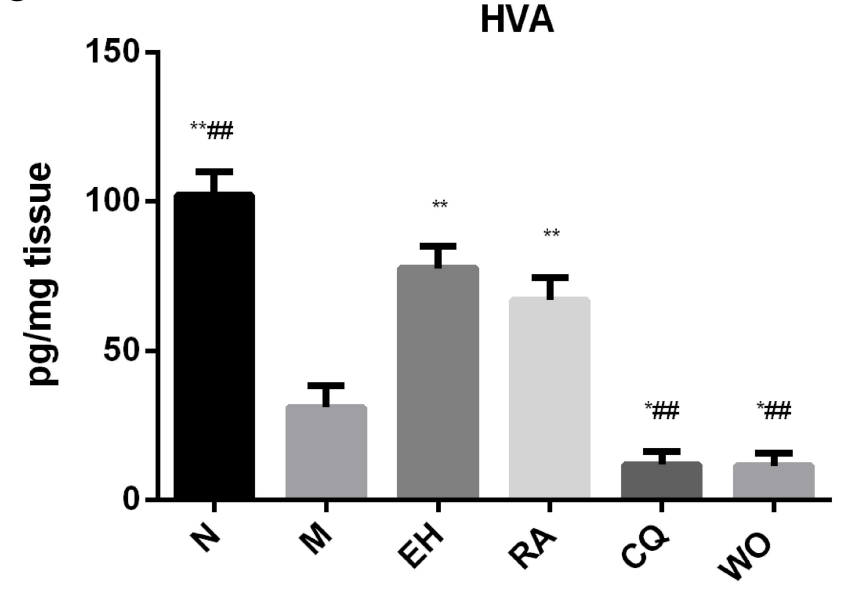

B

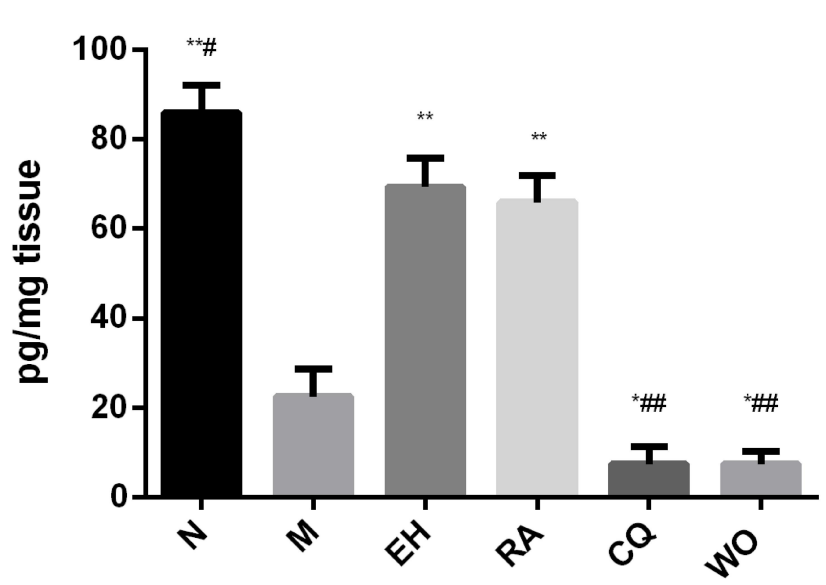

D

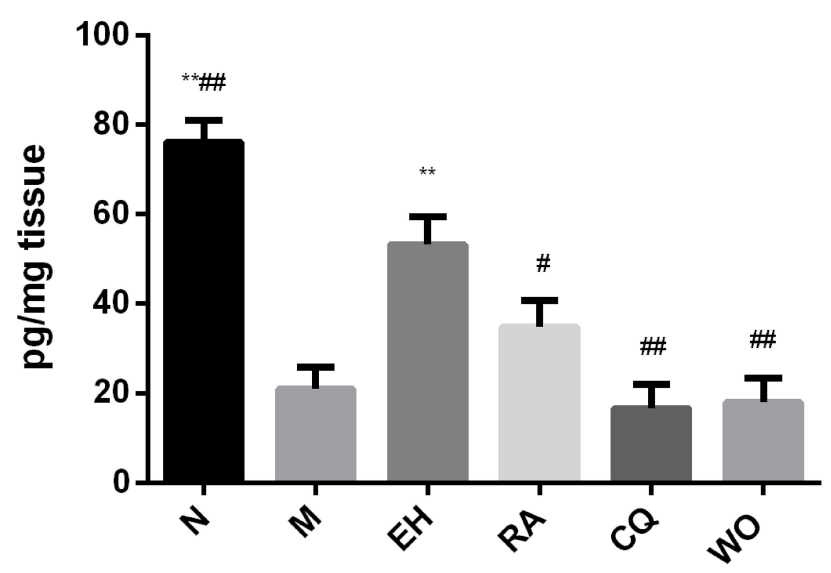

Figure 2 Detection of the neurotransmitter contents in the striatum by the High-performance liquid phase electrochemical method (HPLC-ECD). (A) Dopamine (DA); (B) dihydroxyphenylacetic acid (DOPAC); (C) homovanillic acid (HVA); (D) 5-hydroxytryptamine (5-HT).

Notes: ${ }^{*} \mathrm{P}<0.05$, ${ }^{*} \mathrm{P}<0.0 \mathrm{l}$, compared with the model group; ${ }^{\#} \mathrm{P}<0.05,{ }^{\#} \mathrm{P}<0.0 \mathrm{l}$, compared with the high-dose echinacoside group.

of $\mathrm{P} 62$ in the $\mathrm{M}$ group increased noticeably compared with the $\mathrm{N}$ group $(\mathrm{P}<0.01)$. The protein expressions of $\mathrm{P} 62$ in the EH group and RA group decreased noticeably compared with the $\mathrm{M}$ group, and the differences were statistically significant $(\mathrm{P}<0.01)$. Compared with the EH group, the protein expressions of P62 in the CQ group and WO group increased noticeably $(\mathrm{P}<0.01)$ (as shown in Figure $5 \mathrm{C}$ ). Then, immunofluorescence staining was conducted for P62 and DAPI. It was found that compared with the $\mathrm{N}$ group, the P62 in the substantia nigra increased more obviously in the $\mathrm{M}$ group, the expressions of P62 decreased in the EH group and the RA group, and expressions of P62 increased in the CQ group and the WO group (as demonstrated in Figure 5D).

\section{Echinacoside Increased the Expression of Beclin I and LC3II in the Substantia Nigra in MPTP-Induced PD Mice}

First, Western blot was adopted to determine the protein expression of Beclin 1 in the substantia nigra in mice of each group. It was found that the protein expression of Beclin 1 in the $\mathrm{M}$ group increased significantly compared with the $\mathrm{N}$ group $(\mathrm{P}<0.01)$. However, the increases in protein expression of Beclin 1 in the $\mathrm{EH}$ group and the RA group were more obvious $(\mathrm{P}<0.01)$. Compared with the $\mathrm{EH}$ group and the $\mathrm{M}$ group, the protein expressions of Beclin 1 in the CQ group and the WO group decreased significantly, and the 


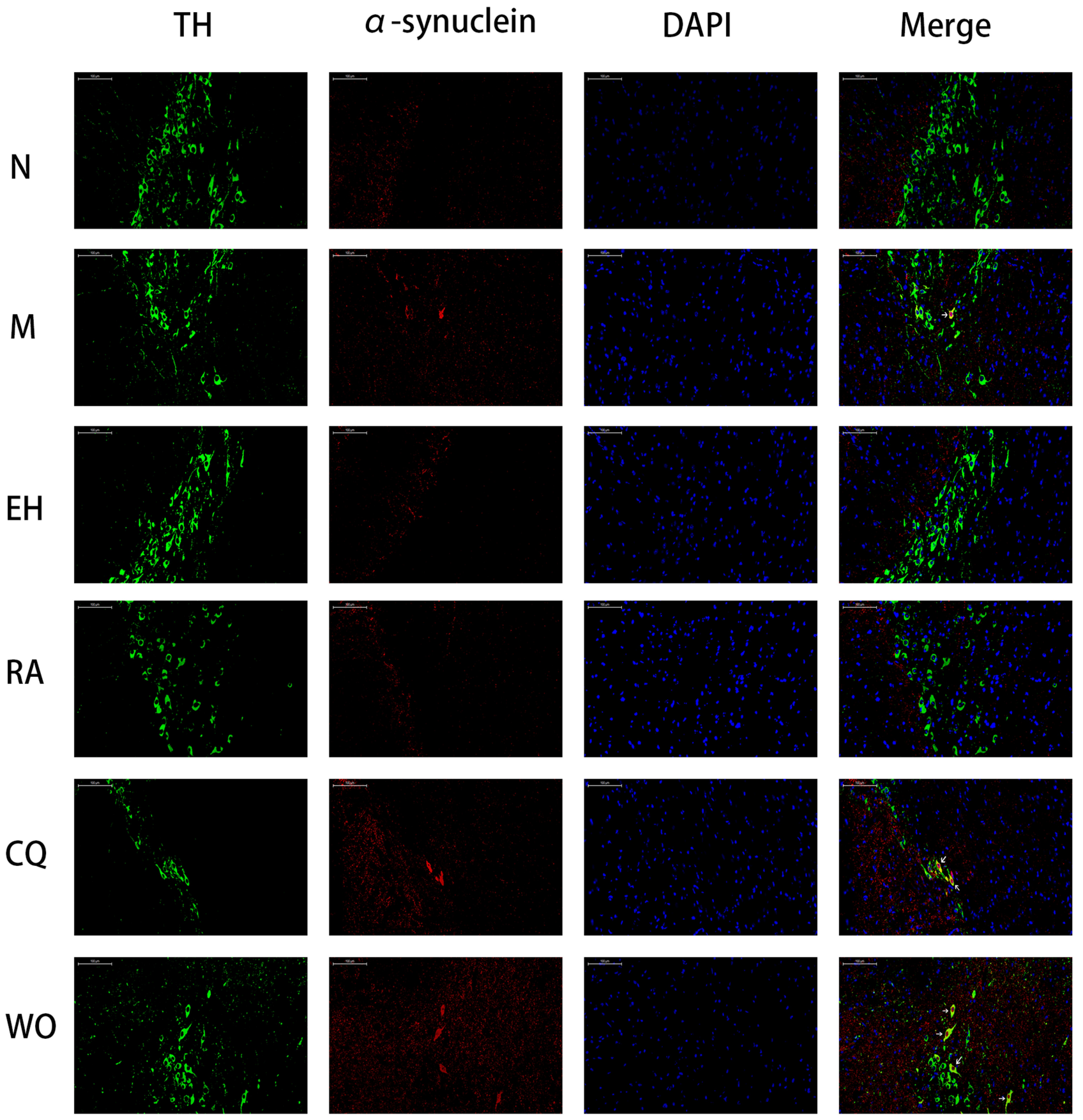

Figure $3 \mathrm{Immunofluorescence} \mathrm{double} \mathrm{staining} \mathrm{of} \mathrm{TH} \mathrm{and} \alpha$-synuclein in the substantia nigra in mice of each group. The figure showed the immunofluorescence double staining of tyrosine hydroxylase $(\mathrm{TH})$ and $\alpha$-synuclein in the substantia nigra in mice of each group, $n=6$, the magnification of the fluorescence microscope: 200x. TH was labeled with green fluorescence, and $\alpha$-synuclein was labeled with red fluorescence, the blue DAPI represented the nucleus. The overlapping of green and red were the double-stained positive cells (yellow).

differences were statistically significant $(\mathrm{P}<0.01)$ (as demonstrated in Figure 5A).

Because LC3 is a key indicator of autophagy, double staining was conducted for $\mathrm{TH}$ and LC3. The results showed that the number of LC3 positive cells in the $M$ group significantly increased compared with that in the $\mathrm{N}$ group. The number of LC3 positive cells in the EH group and the RA group increased more significantly than the $\mathrm{M}$ group. However, when using the autophagy inhibitor, the number of LC3 positive cells in the WO group was significantly lower than that in the $\mathrm{M}$ group, while the number of LC3 positive cells did not decrease 

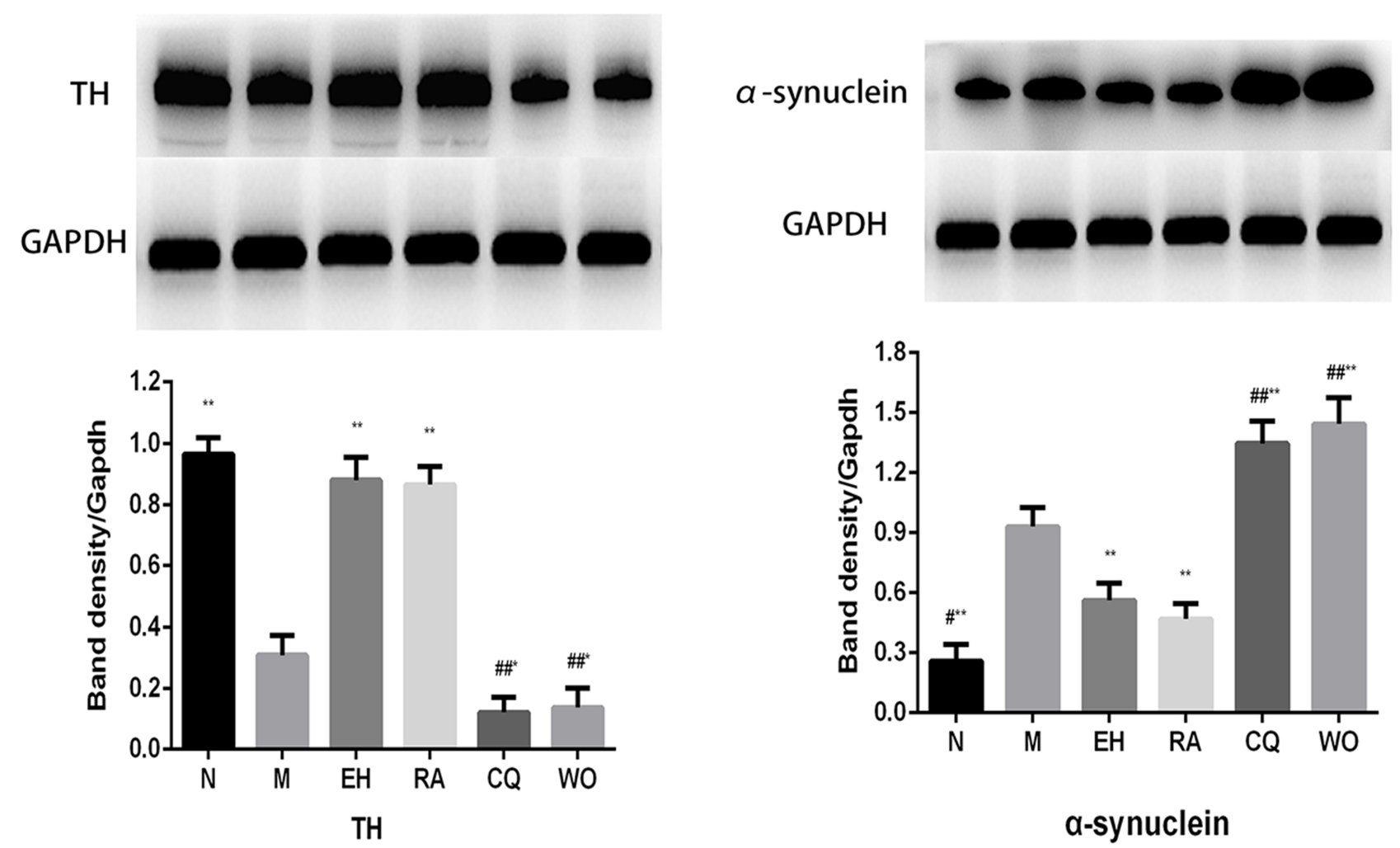

Figure 4 The changes of protein expressions of TH and $\alpha$-synuclein in the substantia nigra in mice of each group.

Notes: ${ }^{*} \mathrm{P}<0.05,{ }^{*} \mathrm{P}<0.01$, compared with the model group; ${ }^{\#} \mathrm{P}<0.05,{ }^{\# \#} \mathrm{P}<0.01$, compared with the high-dose echinacoside group, $\mathrm{n}=6$.

significantly in the CQ group (as shown in Figure 6). Then, Western blot was used to detect the protein expression of LC3 in the substantia nigra in mice of each group. The results indicated that the protein expression of LC3II in the $\mathrm{M}$ group significantly increased compared with the $\mathrm{N}$ group $(\mathrm{P}<0.01)$. Protein expressions of LC3II in the EH group, RA group, and CQ group all increased significantly compared with the $\mathrm{M}$ group $(\mathrm{P}<0.01)$. In the WO group, the protein expression of LC3II decreased compared with the $\mathrm{EH}$ group and the $\mathrm{M}$ group, and the differences were statistically significant $(\mathrm{P}<0.01$ and $\mathrm{P}<0.05$, respectively) (as demonstrated in Figure 5B).

\section{The Changes of the Substantia Nigra Ultrastructure and Autophagosomes Observed by Transmission Electron Microscope}

Through the observation of the substantia nigra ultrastructure, it was found that, except for the $\mathrm{N}$ group, the mice all showed varying degrees of substantia nigra ultrastructure injury, manifested as swelling of mitochondria, expansion of rough endoplasmic reticulum, expansion of Golgi, increase of lysosomes, and forming of autophagosomes with double membrane structure. The number of autophagosomes in the $\mathrm{EH}$ group, RA group, and $\mathrm{CQ}$ group significantly increased compared with other groups. The number of autophagosomes in the CQ group was higher than that in the $\mathrm{M}$ group, which was consistent with the results of an increase in the protein expression of LC3II in the fluorescence double staining and Western blot. It also suggested that the number of LC3II and autophagosomes were correlated to a certain extent. There was no obvious increase in autophagosomes in the WO group, suggesting that in this group, the increase of autophagy caused by echinacoside was inhibited (as demonstrated in Figure 7).

\section{The Effect of Echinacoside on Protein Expression of AKT and $m$ TOR in Substantia Nigra in MPTP-Induced PD Mice}

Western blot was used to detect the protein expression of AKT and mTOR in each group. The results revealed that compared with the $\mathrm{N}$ group, the protein expression of $\mathrm{p}-\mathrm{AKT} / \mathrm{AKT}$ decreased noticeably in the $\mathrm{M}$ group $(\mathrm{P}<0.01)$. The protein 


\section{A}

Beclin 1

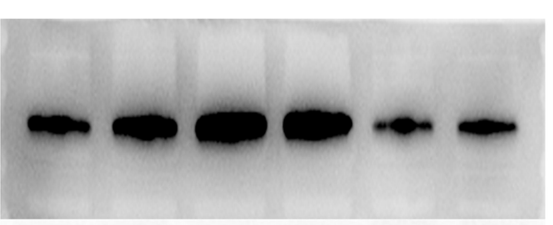

$\beta$-actin

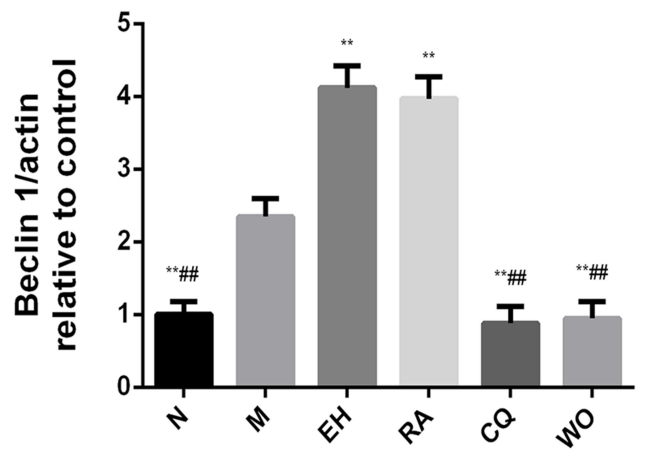

C
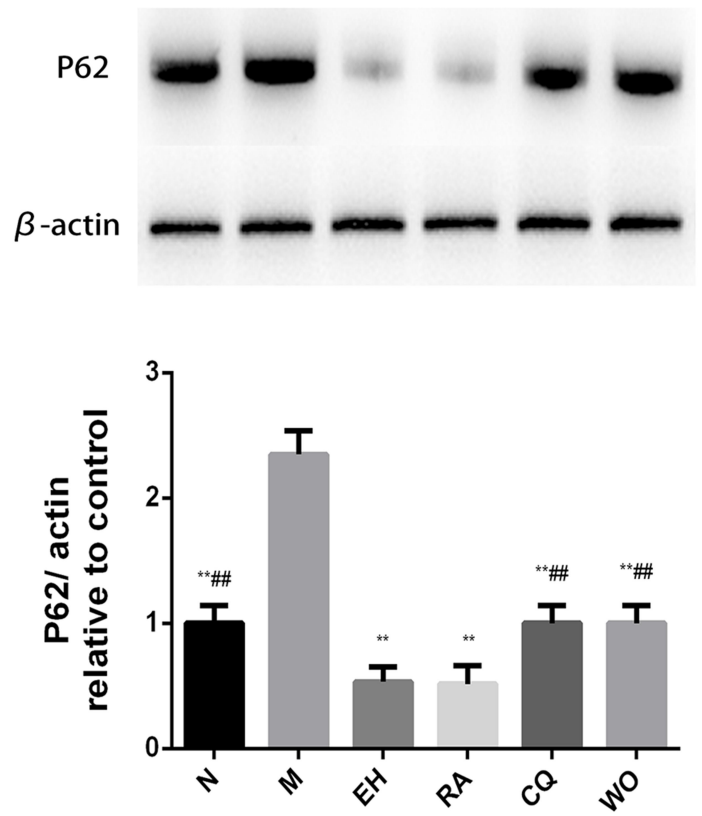

B

LC3- I

LC3- II

\section{$\beta$-actin}

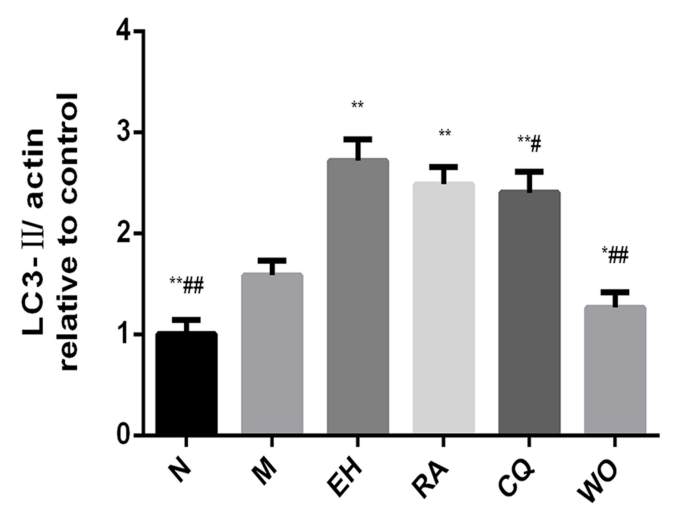

D

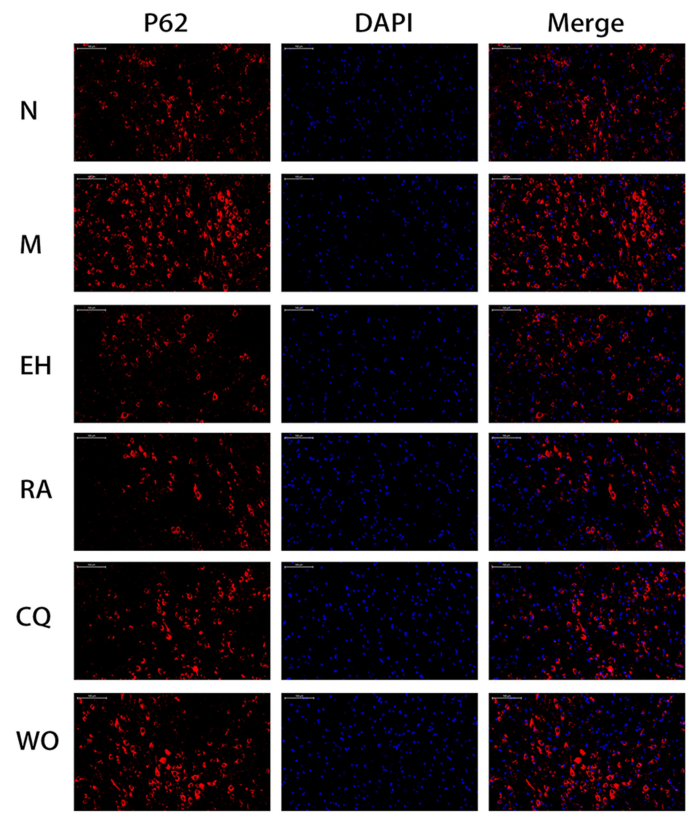

Figure 5 The changes of protein expressions of Beclin I, LC3II, P62, and P62 immunofluorescence staining in the substantia nigra in mice of each group. Notes: $* \mathrm{P}<0.05,{ }^{* * P}<0.01$, compared with the model group; ${ }^{\#} \mathrm{P}<0.05,{ }^{\# \#} \mathrm{P}<0.01$, compared with the high-dose echinacoside group. The magnification of the fluorescence microscope: 200x. P62 was labeled with red fluorescence. The blue DAPI represented the nucleus, $n=6$.

expressions of p-AKT/AKT in the EH group and RA group increased noticeably compared with the $\mathrm{M}$ group $(\mathrm{P}<0.01)$, and the protein expression of $\mathrm{p}-\mathrm{AKT} / \mathrm{AKT}$ in the EH group was higher than that in the RA group $(\mathrm{P}<0.01)$. Compared with the EH group, the protein expressions of $\mathrm{p}-\mathrm{AKT} / \mathrm{AKT}$ in the CQ group and WO group decreased noticeably $(\mathrm{P}<0.01)$ (as shown in Figure 8). Also, it was found that the protein expression of $\mathrm{p}-\mathrm{mTOR} / \mathrm{mTOR}$ in the M group was slightly 

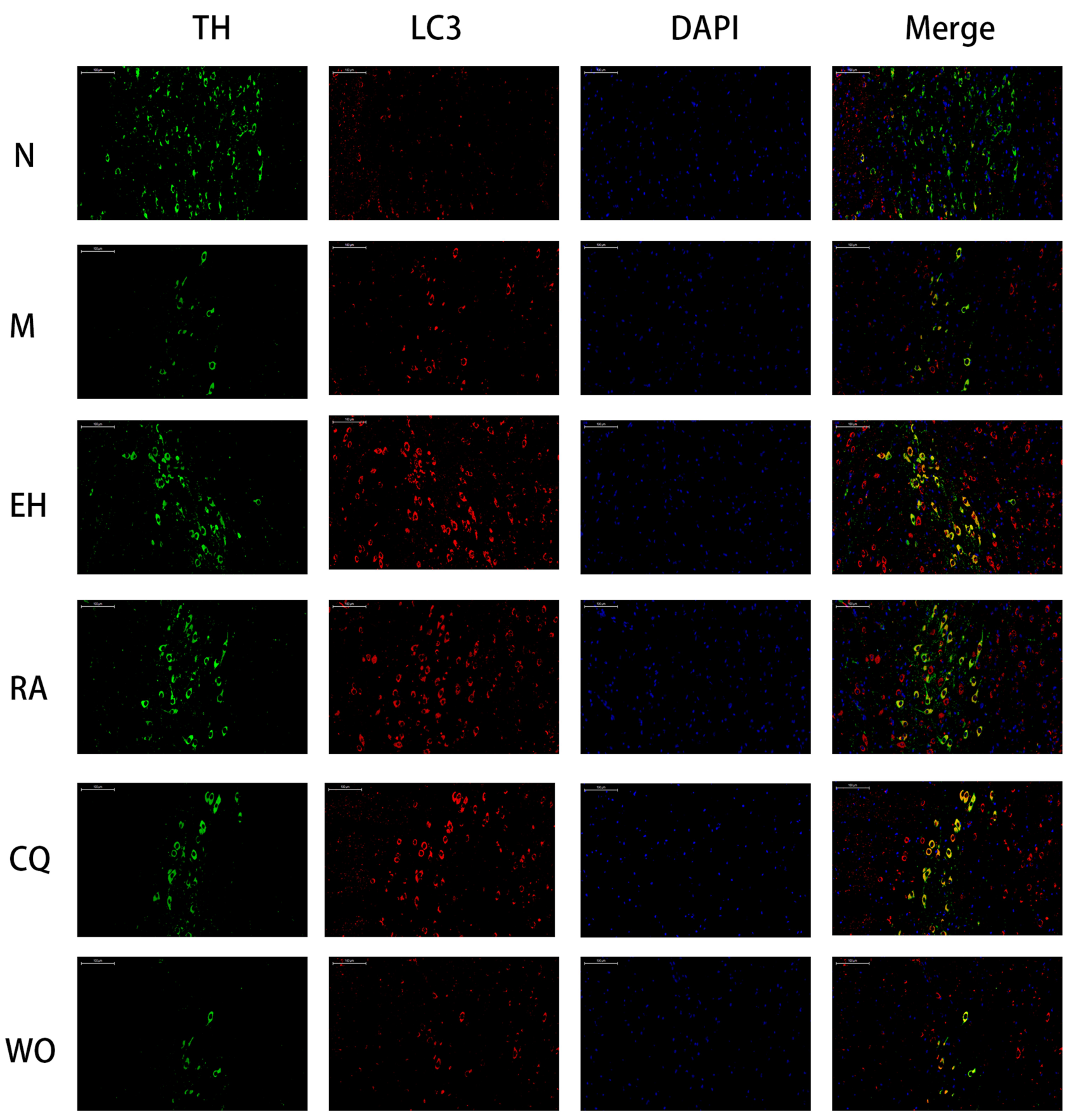

Figure 6 The immunofluorescence staining of TH and LC3 in the substantia nigra in mice of each group. The figure showed the immunofluorescence double staining of TH and LC3 in the substantia nigra in mice of each group, $n=6$; the magnification of the fluorescence microscope: 200x. TH was labeled with green fluorescence, and LC 3 was labeled with red fluorescence. The blue DAPI represented the nucleus. The overlapping of green and red were the double-stained positive cells (yellow).

lower than that in the $\mathrm{N}$ group $(\mathrm{P}<0.05)$, but the decrease in protein expression of $\mathrm{p}-\mathrm{mTOR} / \mathrm{mTOR}$ was more significant in the EH group and the RA group than in the M group (P < 0.01). Compared with the EH group, the protein expression of $\mathrm{p}$-mTOR/mTOR increased in the CQ group and the WO group $(\mathrm{P}<0.01)$ (as demonstrated in Figure 8, the lower part).

\section{Discussion}

The occurrence of PD is related to many factors, but the main pathological mechanisms seem to focus on oxidative stress, mitochondrial dysfunction, and abnormal protein aggregation. All these mechanisms are highly correlated with autophagy. ${ }^{16}$ For living organisms, autophagy plays 

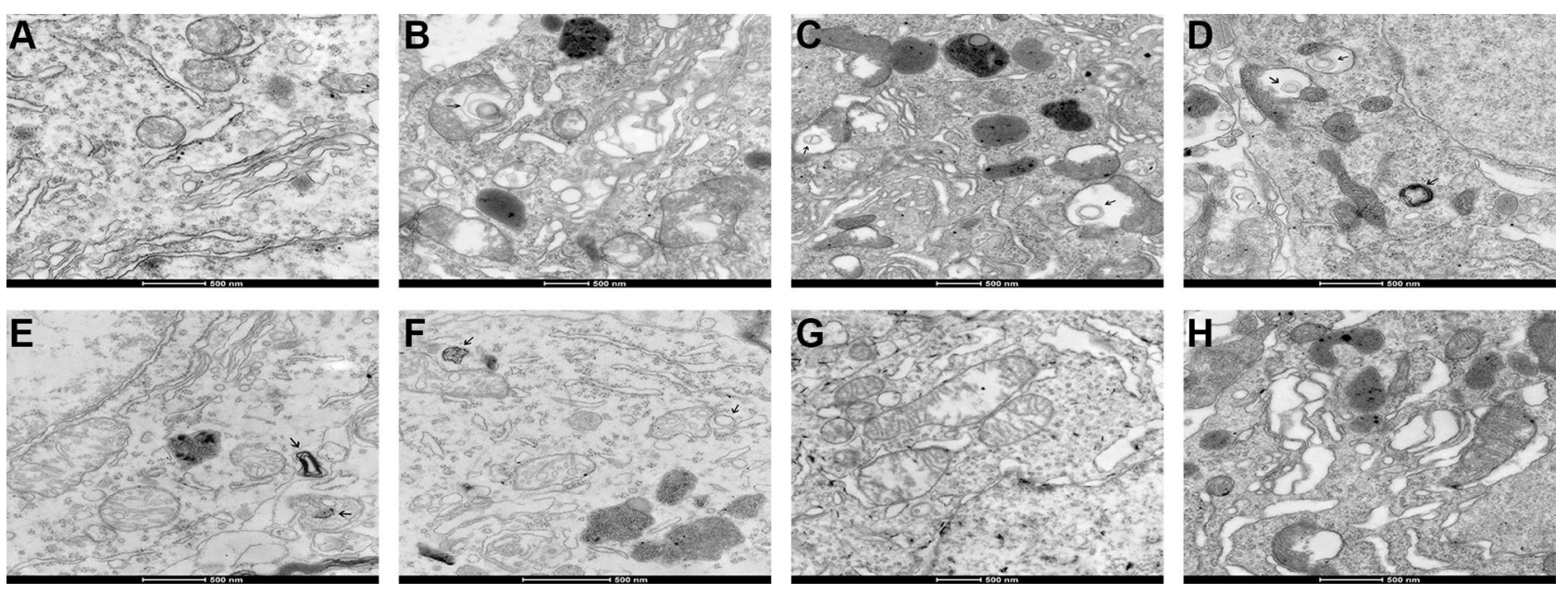

Figure 7 The ultrastructural changes and formation of autophagosome in the substantia nigra in mice of each group. (A) The normal saline control group, with the normal structure of the substantia nigra; (B) the model group, with the manifestations of mitochondria swelling, rough endoplasmic reticulum expansion, and formation of lysosome and autophagosome; (C and D) the echinacoside group, with relatively more formation of lysosome and autophagosome; (E) the rapamycin group, with manifestations of mitochondria swelling and formation of lysosome and autophagosome; (F) the chloroquine group, with manifestations of mitochondria swelling and formation of lysosome and autophagosome; ( $\mathbf{G}$ and $\mathbf{H}$ ) the wortmannin group, with noticeably swollen mitochondria, expanded rough endoplasmic reticulum (G) and swelling of mitochondria, expansion of rough endoplasmic reticulum, obvious expansion of the Golgi and increase of lysosomes $(\mathbf{H})$.

an important role. It plays a key role in removing the misfolded proteins, the abnormally aggregated proteins, and damaged organelles. ${ }^{17}$

It has been reported that the up-regulation of autophagy can eliminate some disease-related proteins in PD, such as $\alpha$-synuclein, ubiquitin, and other misfolded proteins, thereby reducing the incidence of $\mathrm{PD}{ }^{18,19}$ Meanwhile, studies have also pointed out that the down-regulation of autophagy can lead to mitochondrial dysfunction, mitochondrial rupture, deposition of insoluble SNCA/ $\alpha$-synuclein, and ubiquitin proteins. ${ }^{10}$ Therefore, it is important to regulate autophagy to maintain normal function.

Preliminary studies of our research group also confirmed in both Vivo and Vitro studies that ECH had a certain neuroprotective effect ${ }^{9,20-23}$ and may reduce the expression of $\alpha$-synuclein. To further explore the specific mechanism of ECH in the clearance of $\alpha$-synuclein, we conducted a more in-depth study on the neuroprotective mechanism of ECH in MPTP-induced PD mice from the perspective of autophagy.

In the present study, the neurobehaviors in mice of each group were evaluated first. It was found that compared with the $\mathrm{M}$ group, the climbing time of mice in the EH group was significantly shorter, and the latency to fall and the stride length of the fore and hind limbs were significantly extended. The ability of autonomous activity in mice in the EH group was also significantly increased. The results of HPLC showed that the content of monoamine neurotransmitters increased significantly in the EH group compared with the $\mathrm{M}$ group, especially the expression of DA and DOPAC. The results of Western blot also revealed that the expression of $\mathrm{TH}$ in the substantia nigra increased noticeably in the EH group compared with the $\mathrm{M}$ group. While the above neurobehavioral functions deteriorated significantly in the CQ group and WO group, the content of DA and TH were also significantly lower than that in the EH group, and the mortality was significantly higher. According to the results of the present study, the mortality in the autophagy inhibitor groups was higher than in the other groups. The mortality in the CQ group was $28 \%$, and that in the WO group was $12 \%$, while there were no deaths in the other groups. To verify the effect of autophagy inhibitors on $\mathrm{ECH}$, double immunofluorescence of TH and $\alpha$-synuclein staining, together with the protein immunoblotting of $\alpha$-synuclein, were adopted to observe the expression of $\alpha$-synuclein. The results confirmed that ECH did significantly reduce the expression of $\alpha$-synuclein compared with the $\mathrm{M}$ group. However, with the application of autophagy inhibitors, the expression of $\alpha$-synuclein increased sharply. Studies have pointed out that the toxic effects of MPTP are closely related to the appearance of $\alpha$-synuclein because the mice of the $\alpha$-synuclein knockout show a high degree of tolerance to the toxicity of MPTP. ${ }^{24}$ This might indirectly suggest that for the MPTP-induced PD mice models, the higher the expression of $\alpha$-synuclein, the stronger the toxic effect of MPTP on mice. In the present study, the mortality of mice in the autophagy inhibitor group was significantly higher 

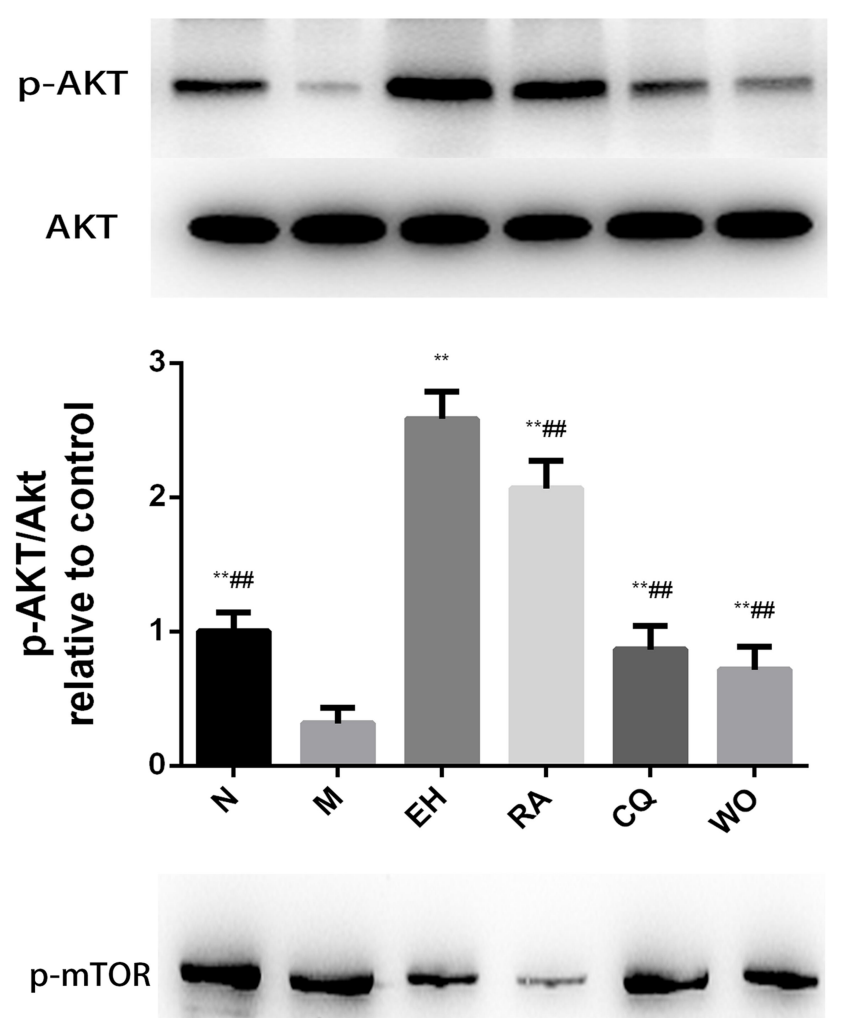

mTOR
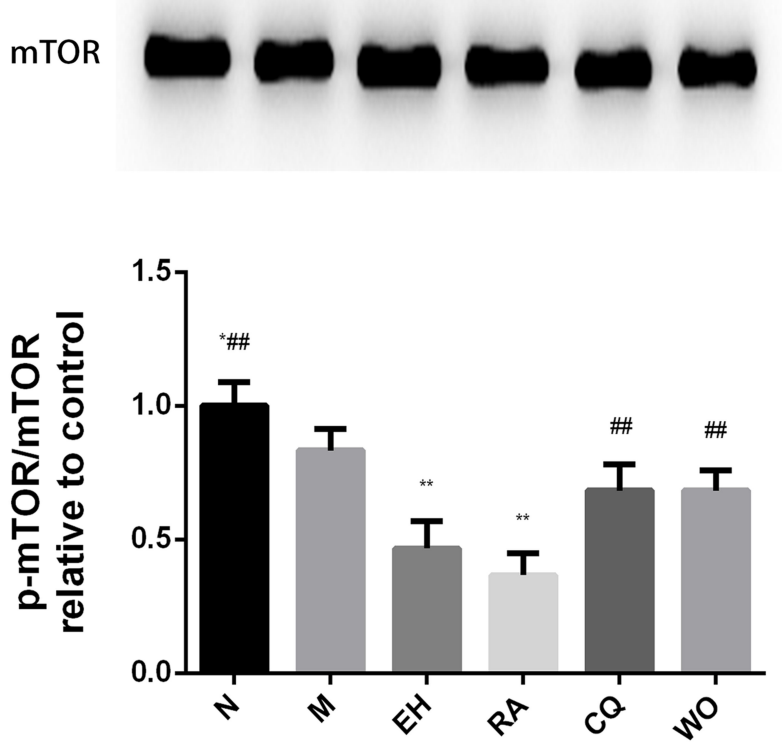

Figure 8 The changes of protein expressions of AKT and mTOR in the substantia nigra in mice of each group.

Notes: $* \mathrm{P}<0.05$, $* * \mathrm{P}<0.01$, compared with the model group; ${ }^{\#} \mathrm{P}<0.01$, compared with the high-dose echinacoside group, $n=6$.

than that in other groups, which might be correlated with the increased expression of $\alpha$-synuclein and the decreased tolerance to the toxic effects of MPTP. Therefore, we inferred that the neuroprotective effect of ECH might be correlated with the promotion of autophagy, and autophagy inhibitors might significantly increase the expression of $\alpha$-synuclein, which might be correlated with the death of mice.

As a key marker of autophagy, the light chain 3 of the microtubule-associated protein 1 (LC3) is widely used to detect the occurrence of autophagy by immunofluorescence or Western blot. P62, also known as SQSTM1, is involved in the pathogenesis of a variety of neurodegenerative diseases. It can link the ubiquitin proteins to LC3 and degrade through autophagy. The protein expression level of P62 can reflect the completion of autophagy flow. ${ }^{25}$ To further investigate the regulation mechanism of ECH on MPTP-induced autophagy in PD mice, the expression of autophagy-related indicators such as Beclin 1, LC3 and P62 were measured in mice in each group.

It was found that the expressions of Beclin1 and LC3II increased significantly in the $\mathrm{M}$ group compared with the $\mathrm{N}$ group, together with an obvious increase in the number of the autophagosome. These suggested that the level of autophagy in mice in the M group was increased. However, the level of P62 did not decrease with the increase of autophagy. Instead, it increased significantly, indicating that the entire autophagy process was not completed normally. That meant there existed an obvious autophagic flow disorder in the MPTP group, which was consistent with reports in the literature. ${ }^{26,27}$ Rapamycin can increase the synthesis of lysosomes and promote the clearance of autophagosomes. In the present study, the classic autophagy inducer rapamycin was adopted as a positive control, and the classic autophagy inhibitors chloroquine and wortmannin were used as controls. Immunofluorescence and Western blot were used to observe the expression level of LC3. The expression of Beclin 1 was measured by Western blot, and the expression of autophagosomes in each group of mice was observed by a transmission electron microscope. The results revealed that compared with the $\mathrm{M}$ group, the expressions of Beclin1 and LC3-II increased in the ECH group and RA group, while the levels of P62 and $\alpha$ synuclein decreased significantly, indicating that in these two groups, the autophagic flow disorder improved significantly than that in MPTP-induced PD mice. The expressions of Beclin 1, LC3-II and the number of autophagosomes in the WO group decreased, accompanied by the increased levels of P62 and $\alpha$-synuclein. This correlated with the failure of clearance of the autophagy substrates and pathological products over time due to autophagy inhibition. The expression of LC3-II and the 
number of autophagosomes in the CQ group increased significantly, along with an increase in the levels of P62 and $\alpha$-synuclein, which was correlated with the disorder of autophagy flow.

It is known that mTOR kinase is a key regulatory site for autophagy, and it exists in the cell as two complexes with different functions, mTORC1 and mTORC2. mTORC1, which is sensitive to rapamycin, is the main negative feedback regulator of autophagy, while mTORC2, which is not sensitive to rapamycin, can regulate the activation of protein kinase AKT. The activated AKT can act on the mTORC1 complex; therefore, AKT may act as the connection point between mTORC1 and mTORC2. ${ }^{28}$ Through the present study, it was found that, like rapamycin, ECH could up-regulate autophagy by inhibiting the expression of mTOR and also up-regulate the expression of p-AKT/AKT. We speculated that ECH might inhibit the expression of mTORC1, thereby up-regulating autophagy, achieving the effect of eliminating $\alpha$-synuclein and P62, and then activating AKT through mTORC2 to promote the survival of dopamine neurons. However, chloroquine is not the inhibitor of mTOR-dependent induction of autophagy and dephosphorylation of the regulator, so it will be avoided in future studies as experimental means for mTOR-dependent inhibition of autophagy.

\section{Summary}

In short, targeted at mTOR, the development of drugs that can both increase the clinical efficacy and reduce clinical toxicity may be the direction of future research. ECH has many advantages, such as safety, non-toxicity, low side effects, and low price. Our preliminary animal experiments had shown that ECH could inhibit the mTOR pathway, increase autophagy, and promote the decrease of P62 and the elimination of pathological products such as $\alpha$-synuclein. This could bring new treatment strategies for traditional Chinese medicine to intervene in PD. However, considering the insufficient sample size and no relevant cell experiments for verification, this would be the direction of our further research.

\section{Ethics Approval and Consent to Participate}

All experiments were evaluated and approved by the ethics Committee of Nanjing Hospital of Chinese Medicine and complied with the National Institutes of Health Guide for the Care and Use of Laboratory Animals (KY2017102).

\section{Acknowledgments}

We are particularly grateful to all the people who have given us help on our article.

\section{Funding}

This study was funded by:1. Nanjing Health Science and Technology Development Special Fund Grant (YKK18134);2. Jiangsu Provincial Bureau of Traditional Chinese Medicine "Chinese Medicine Brain Diseases" Key Discipline Cultivation Project (NBPY201704)3. Nanjing University of Chinese Medicine "Jiangsu University Nursing Advantageous Discipline Construction Project Funding Project" (2019YSHL098);4. Nanjing "Thirteenth Five-Year Plan" Famous Chinese Medicine Doctor (Kong-Jiang Liu) Studio Construction Project (LKJ-2017-NJ).5. Nanjing "Thirteenth Five-Year Plan" Famous Chinese Medicine Doctor (Jing-Qing Wang) Studio Construction Project (WJQ-2019-NJ).The funding body had no role in the design of the study and collection, analysis, and interpretation of data and in writing the manuscript.

\section{Disclosure}

The authors declare that they have no competing interests.

\section{References}

1. Betarbet R, Sherer TB, MacKenzie G, Garcia-Osuna M, Panov AV, Greenamyre JT. Chronic systemic pesticide exposure reproduces features of Parkinson's disease. Nat Neurosci. 2000;3(12):1301-13 06. doi:10.1038/81834

2. Dunnett SB, Björklund A. Prospects for new restorative and neuroprotective treatments in Parkinson's disease. Nature. 1999;399(6738 Suppl):A32-A39. doi:10.1038/399a032

3. Glick D, Barth S, Macleod KF. Autophagy: cellular and molecular mechanisms. J Pathol. 2010;221(1):3-12. doi:10.1002/path.2697

4. Tan CC, Yu JT, Tan MS, Jiang T, Zhu XC, Tan L. Autophagy in aging and neurodegenerative diseases: implications for pathogenesis and therapy. Neurobiol Aging. 2014;35(5):941-957. doi:10.1016/j. neurobiolaging.2013.11.019

5. Webb JL, Ravikumar B, Atkins J, Skepper JN, Rubinsztein DC. Alphasynuclein is degraded by both autophagy and the proteasome. $J$ Biol Chem. 2003;278(27):25009-25013. doi:10.1074/jbc.M300227200

6. Xilouri M, Vogiatzi T, Vekrellis K, Stefanis L. Alpha-synuclein degradation by autophagic pathways: a potential key to Parkinson's disease pathogenesis. Autophagy. 2008;4(7):917-919. doi: $10.4161 /$ auto. 6685

7. Liu K, Shi N, Sun Y, Zhang T, Sun X. Therapeutic effects of rapamycin on MPTP-induced Parkinsonism in mice. Neurochem Res. 2013;38 (1):201-207. doi:10.1007/s11064-012-0909-8 
8. Stoll A, Renz J, Brack A. Isolation and constitutionof echinacoside, a glycoside from the roots of Echinacea angustifolia DC. Helv Chim Acta. 1950;33:1877-1893.

9. Zhang J, Zhang Z, Xiang J. Neuroprotective effects of echinacoside on regulating the stress-active p38MAPK and NF-KB p52 signals in the mice model of Parkinson's disease. Neurochem Res. 2017;42 (4):975-985. doi:10.1007/s11064-016-2130-7

10. Arriola Apelo SI, Pumper CP, Baar EL, Cummings NE, Lamming DW. Intermittent administration of rapamycin extends the life span of female C57BL/6J mice. J Gerontol A Biol Sci Med Sci. 2016;71(7):876-881. doi:10.1093/gerona/glw064

11. Li Y, Wang X, Wei Z, et al. Pretreatment with wortmannin alleviates lipopolysaccharide/d-galactosamine-induced acute liver injury. Biochem Biophys Res Commun. 2014;455(3-4):234-240. doi:10.1016/j. bbrc.2014.10.152

12. Matsuura K, Kabuto H, Makino H, Ogawa N. Pole test is a useful method for evaluating the mouse movement disorder caused by striatal dopamine depletion. J Neurosci Methods. 1997;73(1):45-48. doi:10.1016/s0165-0270(96)02211-x

13. Sedelis M, Schwarting RK, Huston JP. Behavioral phenotyping of the MPTP mouse model of Parkinson's disease. Behav Brain Res. 2001;125(1-2):109-125. doi:10.1016/s0166-4328(01)00309-6

14. Byler SL, Boehm GW, Karp JD, et al. Systemic lipopolysaccharide plus MPTP as a model of dopamine loss and gait instability in $\mathrm{C} 57 \mathrm{Bl} /$ 6J mice. Behav Brain Res. 2009;198(2):434-439. doi:10.1016/j. bbr.2008.11.027

15. Baquet ZC, Gorski JA, Jones KR. Early striatal dendrite deficits followed by neuron loss with advanced age in the absence of anterograde cortical brain-derived neurotrophic factor. $J$ Neurosci. 2004;24(17):4250-4258. doi:10.1523/JNEUROSCI.3920-03.2004

16. Lynch-Day MA, Mao K, Wang K, Zhao M, Klionsky DJ. The role of autophagy in Parkinson's disease. Cold Spring Harb Perspect Med. 2012;2(4):a009357. doi:10.1101/cshperspect.a009357

17. Rubinsztein DC, Bento CF, Deretic V. Therapeutic targeting of autophagy in neurodegenerative and infectious diseases. J Exp Med. 2015;212(7):979-990. doi:10.1084/jem.20150956

18. Dadakhujaev S, Noh HS, Jung EJ, et al. Autophagy protects the rotenone-induced cell death in alpha-synuclein overexpressing SH-SY5Y cells. Neurosci Lett. 2010;472(1):47-52. doi:10.1016/j. neulet.2010.01.053
19. Xiong N, Jia M, Chen C, et al. Potential autophagy enhancers attenuate rotenone-induced toxicity in SH-SY5Y. Neuroscience. 2011;199:292-302. doi:10.1016/j.neuroscience.2011.10.031

20. Zhao Q, Gao J, Li W, Cai D. Neurotrophic and neurorescue effects of Echinacoside in the subacute MPTP mouse model of Parkinson's disease. Brain Res. 2010;1346:224-236. doi:10.1016/j.brainres. 2010.05.018

21. Zhu M, Zhou M, Shi Y, Li WW. Effects of echinacoside on MPP $(+)$-induced mitochondrial fragmentation, mitophagy and cell apoptosis in SH-SY5Y cells. Zhong Xi Yi Jie He Xue Bao. 2012;10 (12):1427-1432. doi:10.3736/jcim20121215

22. Zhao Q, Yang X, Cai D, et al. Echinacoside protects against MPP (+)-induced neuronal apoptosis via ROS/ATF3/CHOP pathway regulation. Neurosci Bull. 2016;32(4):349-362. doi:10.1007/s12264016-0047-4

23. Chen C, Xia B, Tang L, et al. Echinacoside protects against MPTP/ $\mathrm{MPP}+$-induced neurotoxicity via regulating autophagy pathway mediated by Sirt1. Metab Brain Dis. 2019;34(1):203-212. doi:10. 1007/s11011-018-0330-3

24. Dauer W, Kholodilov N, Vila M, et al. Resistance of alpha -synuclein null mice to the parkinsonian neurotoxin MPTP. Proc Natl Acad Sci US A. 2002;99(22):14524-14529. doi:10.1073/pnas.172514599

25. Kabeya Y, Mizushima N, Ueno T, et al. LC3, a mammalian homologue of yeast Apg8p, is localized in autophagosome membranes after processing [published correction appears in EMBO J. 2003 Sep 1;22(17):4577]. EMBO J. 2000;19(21):5720-5728. doi:10.1093/ emboj/19.21.5720

26. Lamine-Ajili A, Fahmy AM, Létourneau M, et al. Effect of the pituitary adenylate cyclase-activating polypeptide on the autophagic activation observed in in vitro and in vivo models of Parkinson's disease. Biochim Biophys Acta. 2016;1862(4):688-695. doi:10.1016/ j.bbadis.2016.01.005

27. Lu M, Su C, Qiao C, Bian Y, Ding J, Hu G. Metformin prevents dopaminergic neuron death in MPTP/P-induced mouse model of Parkinson's disease via autophagy and mitochondrial ROS clearance. Int J Neuropsychopharmacol. 2016;19(9):pyw047. doi:10.1093/ijnp/ pyw047

28. Swiech L, Perycz M, Malik A, Jaworski J. Role of mTOR in physiology and pathology of the nervous system. Biochim Biophys Acta. 2008;1784(1):116-132. doi:10.1016/j.bbapap.2007.08.015
Neuropsychiatric Disease and Treatment

\section{Publish your work in this journal}

Neuropsychiatric Disease and Treatment is an international, peerreviewed journal of clinical therapeutics and pharmacology focusing on concise rapid reporting of clinical or pre-clinical studies on a range of neuropsychiatric and neurological disorders. This journal is indexed on PubMed Central, the 'PsycINFO' database and CAS, and is the official journal of The International Neuropsychiatric Association (INA). The manuscript management system is completely online and includes a very quick and fair peer-review system, which is all easy to use. Visit http://www.dovepress.com/testimonials.php to read real quotes from published authors. 